\title{
Глубинный геоэлектрический разрез по профилю SVEKA по результатам әкспериментов BEAR и FENICS
}

\section{Петрищев М.С.}

Санкт-Петербургский филиал ФГБУН Института земного магнетизма, ионосферы и распространения радиоволн им. Н.В. Пушкова РАН (СПбФ ИЗМИРАН), Санкт-Петербург,.ms_petr@mail.ru

Аннотация. Эксперимент BEAR основан на длительных синхронных магнитотеллурических наблюдениях на сети из 50 станций, расположенных по всему Фенноскандинавскому щиту вдоль регулярной сети $150 \times 150$ км. Его основной задачей было изучение строения астеносферы на глубинах в сотни километров. Обработка и интерпретация были выполнены с использованием 1D и 2D моделирования международной командой. Однако до настоящего времени не было получено общепринятого результата интерпретации. Целью нашего исследования была попытка количественно интерпретировать результаты эксперимента BEAR, используя феноменологический подход. В работе представлен квазидвумерный разрез литосферы на глубину 300 км по профилю SVEKA длиной 800 км.

Ключевые слова: SVEKA, Фенноскандинавский щит, промежуточный проводящий слой.

\section{Deep geoelectric section along the SVEKA profile according to the results of BEAR and FENICS experiments}

\section{Petrishchev M.S.}

Pushkov Institute for terrestrial magnetism, ionosphere and radio wawe propagation of the Russian Academy of sciences, Saint-Petersburg branch,St.Petersburg,ms_petr@mail.ru

\begin{abstract}
The BEAR experiment technique is based on a long-term synchronous magnetotelluric observations at a network of 50 stations located throughout the Fennoscandian Shield along a regular network of $150 \times 150 \mathrm{~km}$. Its main task was to study the structure of the asthenosphere at depths of hundreds of kilometers. Processing and interpretation was carried out using 1D and 2D modeling by international team. However, to date, no generally accepted interpretation result has been received. The aim of our study was an attempt to quantitatively interpret the results of the BEAR experiment using a phenomenological approach. The quasi-two-dimensional section of the lithosphere to a depth of $300 \mathrm{~km}$ along the SVEKA profile of $800 \mathrm{~km}$ long is elaborated and discussed.
\end{abstract}

Key words: Fennoscandian Shield; intermediate conductive layer; SVEKA.

\section{Введение}

Считается, что древние кристаллические щиты являются наиболее благоприятными областями для изучения глубинного строения литосферы с использованием электромагнитных методов. Отсутствие осадочного чехла и высокое удельное сопротивление кристаллических пород является основной причиной такого суждения. Фенноскандинавский (Балтийский) щит площадью около 1 миллиона квадратных километров является идеальным объектом в этом отношении. История глубоких геоэлектрических исследований на Балтийском щите начинается с первого эксперимента по глубинному зондированию, проведенного в водах Финского залива и на Карельском перешейке. $\mathrm{C}$ тех пор на протяжении более 70 лет возможности и задачи электромагнитного зондирования значительно расширились благодаря совершенствованию методов наблюдения и теории интерпретации. Эксперимент BEAR (Baltic Electromagnetic Array Research) был одним из этапов развития в этом направлении. Поиск возможного появления астеносферы, представляющей собой промежуточный проводящий слой частично расплавленных пород на глубинах в несколько сотен километров, был принят в качестве основной цели эксперимента BEAR. Идея и методика эксперимента были основаны на проведении синхронных магнитотеллурических зондирований (МТЗ) в единой сети наблюдений для последующей двухмерной и трехмерной интерпретации, поскольку отдельные МТЗ, которые ранее использовались для этой цели, не дали положительных результатов из-за 


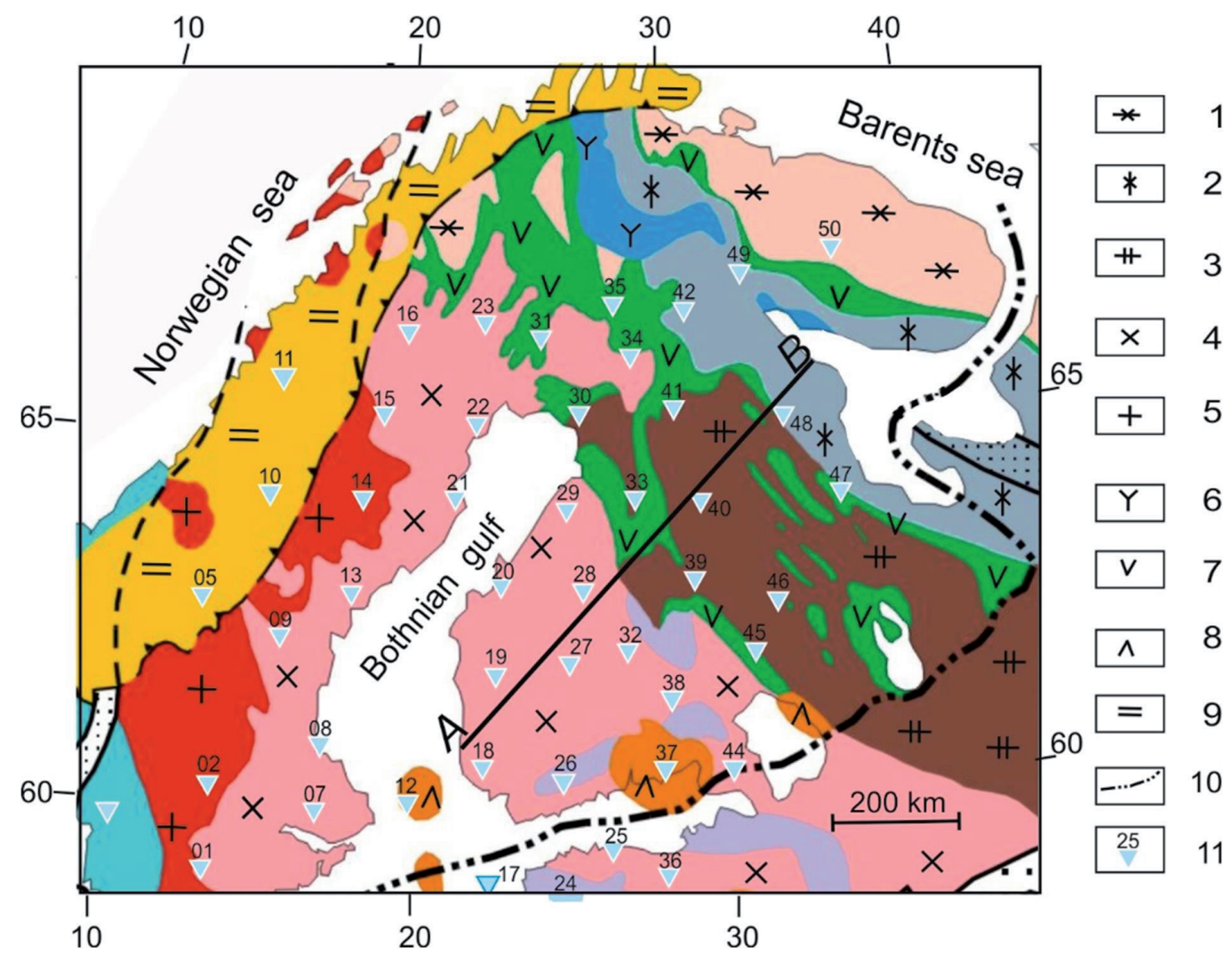

Рис. 1. Расположение измерительных станций (треугольники 11) в эксперименте «BEAR» на геологической основе (Glaznev, 2003). Сплошная линия $A B$ показывает положение профиля SVEKA.

Архейские блоки: 1 -мурманский, 2-беломорский, 3-карельский; протерозойские блоки: 4-фенноскандинавский, 5 - готский; вулканогенно-осадочные комплексы: 6 - гранулиты, 7 - зеленокаменные пояса, 8 - рапакивиграниты; 9 - каледониды; 10 - граница между Феннискандинавским щитом и Русской платформой.

Fig. 1. The layout of the measuring stations in the BEAR experiment (triangles 11) and their numbers on a geological sketch (Glaznev, 2003) and position of the SVEKA profile (solid line).

Archaean blocks: 1 - Murmansk, 2 - White Sea, 3 - Karelian; Proterozoic blocks: 4 - Fennoscandian, 5 - Gothnian; Volcanic rocks: 6 - Granulites, 7 - Greenstone belts, 8 - Rapakivi granites; 9 - Caledonides; 10 - the boundary between the Fennoscandian Shield and Russian platform.

влияния горизонтальной неоднородности среды и неоднородности источника естественных вариаций электромагнитного поля Земли (Rokityansky et al., 1963).

Результаты работы над проектом BEAR представлены в ряде статей, однако единый подход к анализу данных и, соответственно, общепринятый результат интерпретации пока не получен. Основной причиной была большая, в основном нерегулярная, вариация значений кажущегося удельного сопротивления. Указанный разброс значений сокращается при применении феноменологического подхода (Zhamaletdinov \& Petrishchev, 2015). Далее кратко остановимся на сути указанного подхода применительно к данным эксперимента BEAR и выполним анализ этих материалов по профилю SVEKA.

\section{1. Интерпретация данных электромагнитных зондирований BEAR в рамках феноменологического подхода}

Феноменологический подход основан на использовании регуляризирующих параметров (постулатов), априорных ограничений, установленных на основе предыдущих результатов электромагнитных зондирований, в основном с использованием мощных управляемых источников (Zhamaletdinov, 2011). Использованы следующие постулаты: так называемые «коровые» проводящие ано- 
малии распределены исключительно внутри верхнего слоя земной коры толщиной 10 км; верхний неоднородный слой земной коры толщиной 10 км расположен на поверхности горизонтально однородного слабопроводящего слоистого фундамента; средняя продольная электропроводность верхнего слоя земной коры толщиной 10 км на территории Фенноскандского щита составляет 1 См. Применение такого подхода позволило построить квазитрехмерную модель электропроводности литосферы Фенноскандинавского щита (Zhamaletdinov \& Petrishchev, 2015). Положение измерительных станций в эксперименте BEAR и профиля SVEKA представлено на рисунке 1.

\section{2. Интерпретация данных электромагнитных зондирований по профилю SVEKA}

Первые магнитотеллурические наблюдения по профилю SVEKA были сделаны в начале 80-х годов (Adam et al., 1982). Позже, с 1985 г., работа по профилю была продолжена финскими геофизиками. Длина профиля достигла 750 км, а количество зондирований увеличилось до 150 пунктов (Korja et al., 2006). Профиль пересекает несколько палеопротерозойских комплексов Свекофеннского блока и в северо-восточной части переходит в карельский район распространения архейских гранитогнейсов. В средней части, между участками 400 и 500 км, профиль SVEKA пересекает Ладожско-Ботническую зону.

Сводная диаграмма с результатами интерпретации данных BEAR по профилю SVEKA показана на рисунке 2 , где три панели рисунка отражают свойства неоднородного верхнего слоя земной коры толщиной около 10 км.

Гранитоидный комплекс Центральной Финляндии (ЦФГК) и архейские кристаллические породы Кухмо и Карельского блоков земной коры (рис. 2 a) отличаются высокими значениями удельного сопротивления - до $10^{5}$ Ом·м. Пониженные значения удельного сопротивления $\left(10^{2}-10^{3} \mathrm{OM} \cdot \mathrm{M}\right.$
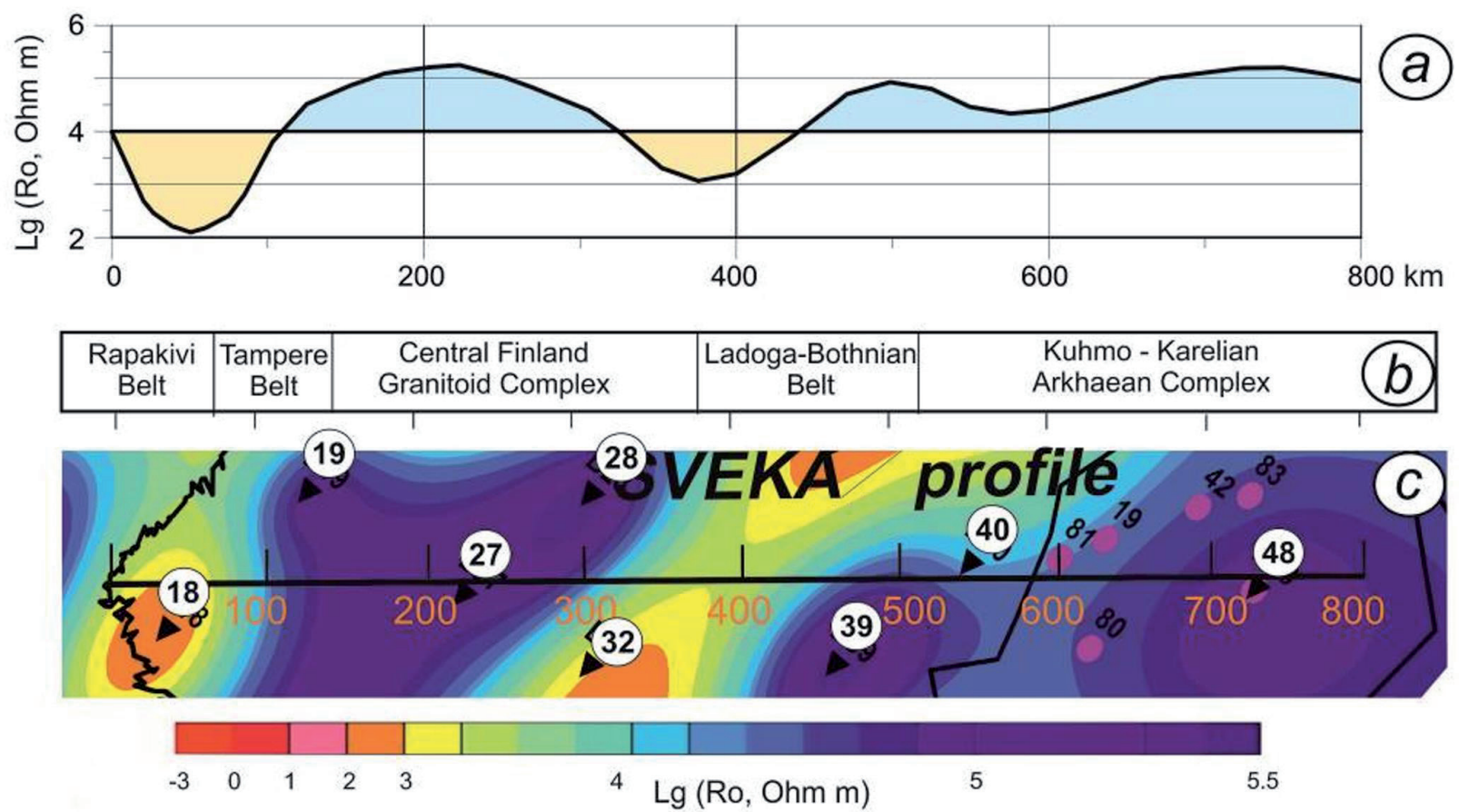

Рис. 2. Электропроводность верхней 10-километровой толщи земной коры на профиле SVEKA по результатам интерпретации данных BEAR.

a - график электрического сопротивления земной коры в пределах верхнего 10-километрового слоя, б - названия геологических блоков, пересекаемых профилем SVEKA; с - план изолиний удельного сопротивления по профилю SVEKA с указанием положения станций эксперимента BEAR (в кружках).

Fig. 2. Electrical conductivity of the upper $10 \mathrm{~km}$ part of the SVEKA profile from results of the BEAR data interpretation. $\mathrm{a}$ - graph of electrical resistivity of the upper 10-km layer of the Earth's crust; $b$ - the legend of geological blocks by the SVEKA profile; $\mathrm{c}$ - plan of isolines of resistivity along the SVEKA profile with the position of BEAR stations (in circles). 


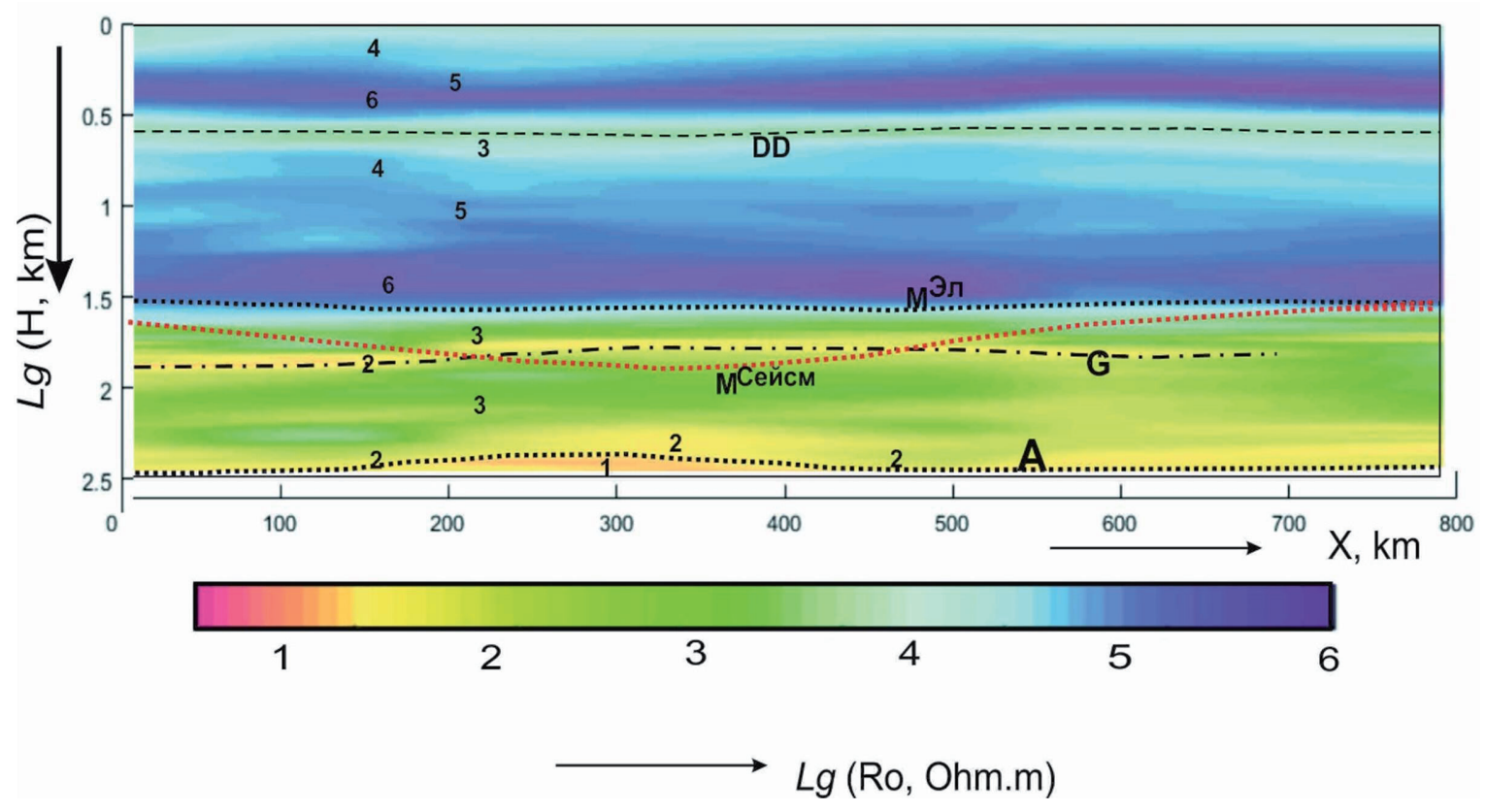

Рис. 3. Квазидвумерный глубинный геоэлектрический разрез по данным BEAR на профиле SVEKA. DD - слой дилатантно-диффузионной проводимости, $\mathrm{M}^{\ni л ~-~ г р а н и ц а ~ М о х о ~ п о ~ д а н н ы м ~ г е о э л е к т р и к и, ~ М ' е и ̆ с м ~}$ - граница Мохо по данным сейсморазведки, G - слой Гутенберга, А - кровля предполагаемой астеносферы. Цифрами показано удельное сопротивление в $\lg \left(\operatorname{Ro}, \mathrm{OM}^{*} \mathrm{M}\right)$. Положение профиля показано на рисунке 1.

Fig. 3. Quasi-two-dimensional deep geoelectrical section from the BEAR data on the SVEKA profile.

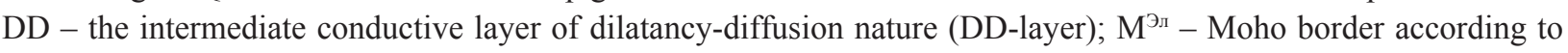
geoelectrics; М Сейсм - Moho border according to seismic data; G - intermediate conductive layer, correlated with Gutenberg boundary from seismic data, A - gypothetical asthenosphere boundary. Black digits 1-6 show resistivity in $\lg (\rho, \Omega \cdot m)$. Location of the profile is shown in Fig. 1.

и ниже) наблюдаются в поясах Рапакиви, Темпере и Ладожско-Ботнической зоне. Легко видеть, что контуры аномалий удельного сопротивления (рис. 2 a) не совсем совпадают с геологическими границами, показанными на рисунке 2b. Различия могут быть объяснены ограниченными возможностями интерполяции данных эксперимента BEAR с редкой сети наблюдений (150-150 км) при решении обратной задачи.

Квази-двумерный разрез по профилю SVEKA (рис. 3) построен на основе одномерной интерпретации данных станций BEAR, приведенных на рисунке 2c. На рисунке 3 видно, что литосфера может быть разделена на две части. Верхняя часть (земная кора) характеризуется средним высоким удельным сопротивлением $\left(10^{4}-10^{6}\right.$ Ом·м) с резким падением удельного сопротивления до $10^{3}$ Ом·м на уровне границы Мохо. В районе глубины 3-5 км выделяется промежуточный проводящий слой дилатантно-диффузионной (флюидной) природы (DD-слой) в виде узкой зоны пониженного удельного сопротивления, порядка $10^{4}$ Ом·м. Впервые слой DD был обнаружен на блоке ЦФГК при проведении частотных зондирований (CSAMT) (Zhamaletdinov et al., 1998). Впоследствии его существование было подтверждено многочисленными наблюдениями CSAMT в восточной части Балтийского щита и, в частности, в Ковдорском и Енско-Беломорском блоках (Zhamaletdinov et al., 2017).

Верхняя мантия, наблюдаемая в диапазоне глубин 40-300 км, характеризуется пониженным удельным сопротивлением $\left(10^{2}-10^{3}\right.$ Ом·м). На этом фоне выделяются две проводящие области. Одна из них, в диапазоне глубин 80-100 км, является прерывистой и имеет удельное сопротивление до 10 Ом·м. Этот слой может быть связан с границей Гутенберга. Вторая проводящая область относится к основанию литосферы на глубине около 300 км. Она может быть связана с наличием гипотетической астеносферы. Удельное сопротивление в пределах предполагаемой астеносферы падает до 10-100 Ом.м. В интервале пикетов 150-450 км выделяется широкая зона слабого 
подъема астеносферного проводящего слоя до уровня 250-280 км с соответствующим увеличением его проводимости. На дневной поверхности этот интервал пикетов соответствует ЦФГК и части Ладожско-Ботнической зоны (рис. 2). Важно отметить, что погружение границы Мохо на глубину 50-60 км (Pavlenkova, 2006; Sharov \& Mitrofanov, 2014) появляется в диапазоне пикетов 150-450 км и, по-видимому, его природа может быть связана с небольшим поднятием астеносферы, отмеченным на рисунке 3.

\section{Выводы}

На профиле SVEKA установлены следующие особенности строения литосферы. Земная кора, вплоть до границы Мохо, характеризуется высоким сопротивлением $10^{5}-10^{6}$ Ом м. В интервале глубин 80-100 км наблюдается прерывистый промежуточный проводящий слой, коррелируемый с сейсмической границей Гутенберга. В основании верхней мантии, на глубине около 300 км установлено существование гипотетической астеносферы. В интервале пикетов 200-400 км наблюдается подъем гипотетической астеносферы до 250 км по снижению удельного сопротивления вещества до 10 Ом·м. Примерно в этом же интервале пикетов установлено погружение границы Мохо до 55-60 км.

Работа выполнена при финансовой поддержке РФФИ, проект № 18-05-00528, а также в рамках госзадания Министерства образования и науки РФ - тема ГИ КНЦ РАН № 0226-2019-0052 и тема ЦЭС КНЦ РАН № 0226-2019-0067.

\section{Литература}

1. Adam A., Kaikkonen P., Hjelt S.E., Pajunpaa K., Szarka L.,Vero J., Wallner A. Magnetotelluric and audiomagnetotellurics measurements in Finland // Tectonophysics. 1982. V. 90. P. 77-90.

2. Glaznev V.N. Complex geophysical models of a lithosphere of the Fennoscandian // Apatity. «KaeM» publishing. 2003. $252 \mathrm{p}$.

3. Korja T., Lahti I. and Pedersen L. The core conductive structure along the SVEKA profile in the central part of the Baltic Shield // In the book: The structure and dynamics of Eastern Europe. Release 2. Moscow. Geocards Geos. 2006. P. 113-121.

4. Pavlenkova N.I. Structure of the lithosphere of the Baltic Shield according to the DSS / Struktura litosfery Baltiiskogo Shchita po dannym GSZ. Structure and Dynamics of the Lithosphere of Eastern Europe. Moscow. Geokart, GEOS. 2006. (in Russian).

5. Rokityansky I.I., Zybin K.Yu., Rokityanskaya DA, Shchepetnev R.V. Magnetotelluric study of the array at the Borok, Lovozero and Petropavlovsk-Kamchatsky geophysical stations // Electromagnetic sounding and magnetotelluric methods of reconnaissance. Leningrad: Leningrad State University. 1963. P. 124-130.

6. Sharov N.V., and Mitrofanov F.P. High-speed heterogeneity of the lithosphere of the Fennoscandian (Baltic) shield / Skorostnye neodnorodnosti litosfery Fennoskandinavskogo Shchita // Reports of the Academy of Sciences. 2014. N. 454 (2). P. 221-224. (in Russian).

7. Zhamaletdinov A.A. The New Data on the Structure of the Continental Earth crust Based on the Results of Electromagnetic with the Use of Powerful Controlled Sources // Doklady Earth Sciences. 2011. V. 438. Part 2. P. 798-802.

8. Zhamaletdinov A.A., Shevtsov A.N., Tokarev A.D., Korja T., Pedersen L. Experiment on the Deep Frequency Sounding and DC Measurements in the Central Finland Granitoid Complex // Electromagnetic Induction in the Earth. 14-th Workshop in Sinaia (Romania). 1998. P. 83.

9. Zhamaletdinov A.A., Petrishchev M. S. Three_Dimensional Model of ithosphere Electrical Conductivity of the Fennoscandian Shield Based on the Results.of the BEAR and FENICS Experiments // Doklady Earth Sciences, 2015. V. 463. Part 1. P. 751-756. DOI: 10.1134/S1028334X15070235.

10. Zhamaletdinov A.A., Velikhov E.P., Shevtsov A.N., Kolobov V.V., Kolesnikov V.E., Skorokhodov A.A., Korotkova T.G., Ivonin V.V., Ryazantsev P.A. and Biruly M.A. The Kovdor-2015 Experiment: Study of the Parameters of a Conductive Layer of Dilatancy-Diffusion Nature (DD Layer) in the Archaean Crystalline Basement of the Baltic Shield // Doklady Earth Sciences. 2017. V. 474. Part 2. P. 641-645. 